\title{
Variabilidad genotípica y ambiental del peso, tamaño y aceite del grano de girasol (Helianthus annuus L.) en el Noreste argentino
}

\author{
Rocca, Paola ${ }^{1}$; Martino, L. ; Rausch, Analia ${ }^{2}$; Zuil, Sebastián G. ${ }^{3}$ \\ ${ }^{1}$ Universidad Nacional de Villa Maria, Córdoba. E-mail: pao_ticino@hotmail.com; laureano_luca@hotmail.com \\ ${ }^{2}$ INTA AER Ceres, Santa Fe. E-mail: rausch.analia@inta.gob.ar \\ ${ }^{3}$ INTA EEA Reconquista, Reconquista, Santa Fe, Argentina E-mail zuil.sebastian@inta.gob.ar
}

\begin{abstract}
RESUMEN
La relación tegumento/embrión es uno de los principales factores que afectan el porcentaje de aceite en girasol. Mayores proporciones de embrión incrementa la cantidad de aceite del grano. En la actualidad, no es claro el efecto ambiental y la variabilidad genotípica en híbridos modernos de girasol de la relación tegumento/embrión y el porcentaje de aceite del grano. Los objetivos de este trabajo fueron i) determinar la variabilidad genotípica y ambiental para porcentaje de aceite y proporción de tegumento y embrión en el Noreste Argentino y ii) analizar la influencia de variables ambientales sobre la relación de tegumento/embrión y el porcentaje de aceite del grano de girasol. Se realizaron 4 experimentos durante la campaña 2014/2015 en las localidades de Reconquista (en dos fechas de siembra), Ceres y Villa Ocampo. Los tratamientos consistieron en una combinación de cuatro ambientes y quince híbridos comerciales de girasol. Las determinaciones realizadas fueron tipo de grano, tamaño (largo y ancho), peso de grano, peso y espesor del tegumento, peso del embrión, contenido de aceite de embrión y grano. Se realizaron análisis de componentes principales para explicar las relaciones de cada componente con las variables medidas. El porcentaje de aceite varió entre 55,2 y 43,3, mientras que el porcentaje de aceite de embrión varió entre 53,5 y 66,4\%. En el porcentaje de aceite en el grano y relación tegumento/ embrión se observaron diferencias significativas entre híbridos y ambientes. El rango obtenido en peso de grano fue de 29 a $91 \mathrm{mg}$. Uno de los factores ambientales más importantes que se relacionó con las variables fue la temperatura máxima y media durante el llenado de granos. Estos resultados contribuyen a la toma de decisiones sobre estabilidad y adaptación genotípica como así también herramienta para el mejoramiento genético para incrementar el porcentaje de aceite de futuros híbridos.
\end{abstract}

Palabras clave: calidad, relación tegumento/embrión, comportamiento genotípico, largo y ancho de grano.

\section{SUMMARY}

The coat/embryo ratio is one of the main factors affecting the percentage of sunflower oil. Higher proportions of seed embryo increase the amount of oil in grain. Currently, environmental effect and genetic variability in modern hybrid sunflower coat/embryo ratio and the percentage of grain oil is unclear. The objectives of this study were i) determine the genotypic and environmental variability for oil percentage and ratio of seed coat and embryo in the northeastern regions of Argentina and ii) analyze the influence of environmental variables on the coat/embryo ratio and oil content sunflower grain. Four experiments during the 2014/2015 growing season were made in Reconquista (in two planting dates), Ceres and Villa Ocampo. The treatments consist in a combination of four environmental conditions by fifteen sunflower commercial hybrids. The determinations were grain type, size (length and width), whole grain weight, weight and thickness of the seed coat, embryo weight and oil content of embryo. Principal component analyses were performed to explain the relationships of each component with the measured variables. The percentage of oil ranged from 55,2 and 43,3\%, while the percentage of seed embryo varied from 53,5 to $66,4 \%$. The grain oil percentage and coat/embryo ratio showed significant differences among hybrids and ambient. The range obtained for grain weight ranged from 29 to $91 \mathrm{mg}$. One of the most important environmental factors related to the variables was maximum and mean temperature during grain filling. These results contribute to decide on adaptation and genotypic stability as well as a tool for breeding programs to increase the foil percentage of future hybrids.

Key words: quality, coat/embryo ratio, genotypic performance, grain length and width. 


\section{INTRODUCCIÓN}

El girasol se considera uno de los cultivos oleaginosos más importantes por poseer gran cantidad de aceite con alto valor nutricional (Sharma et al., 2008). El grano de girasol contiene entre 42 y $55 \%$ de aceite, siendo el embrión donde se deposita la mayor concentración de aceite (entre 57-67\%; López Pereira et al., 2006, Connor y Sadras, 1992). El porcentaje de aceite final del grano dependerá de la tasa de síntesis de aceite diaria y por la duración del llenado efectivo de granos (Aguirrezábal et al., 1996). El embrión representa el $75-80 \%$ del peso seco de la semilla y en él se encuentran dos cotiledones donde se almacenan lípidos, hidratos de carbono y proteínas. El $20-25 \%$ restante del peso seco corresponde al tegumento (López Pereira et al., 2006).

La proporción del pericarpio que es ocupada por el embrión determina la relación tegumento/embrión y está principalmente asociado al componente genético de la semilla (Aguirrezábal y Pereyra, 1998). La relación tegumento/embrión, la aptitud al descascarado y el contenido de ceras se consideran atributos de gran importancia en la cadena agroindustrial. La facilidad del fruto para el descascarado depende de la relación tegumento/embrión y de las características anatómica de los mismos (Aguirrezábal y Pereyra, 1998). Mientras menor sea la proporción del pericarpio con respecto al embrión, menor será la eficacia para el descascarado del mismo debido a que parte del pericarpio queda adherido al embrión (López Pereira et al., 2006). Con el mejoramiento genético, al aumentar el contenido de aceite en la semilla del girasol, se provocó una reducción de la relación tegumento/embrión y una disminución en el espesor del pericarpio, generando dificultades para los procesos industriales de extracción de aceite al que se somete el grano (Figueiredo et al., 2011).

Por otro lado, el tamaño del fruto varía según el tipo de híbrido, condiciones ambientales de crecimiento y ubicación en el capítulo. Los granos de la periferia son más grande que los centrales debido a que la floración es centrípeta, es decir, inicia desde la parte exterior del capítulo hacia el interior del mismo (Santalla et al., 2002; Rondanini et al., 2007, 2009). Aumentos en la fuente disponible durante el llenado de granos generan incrementos en el tamaño del grano pero dependerá del tamaño final del tegumento (Dosio et al., 1997). Estudios recientes indicaron una estrecha relación entre la tasa de crecimiento del tegumento y el tamaño final de grano (Lindstrom, Hernandez, 2015; Castillo et al., 2017). Asimismo, las diferencias en peso de grano entre sectores dentro del capítulo está principalmente debida a la tasa (Connor y Hall, 1997) y/o duración (Chimenti et al., 2001). No obstante, no es clara la relación entre la forma del fruto, las proporciones de tegumento y embrión como tampoco los efectos genotípicos y ambientales en los híbridos modernos de girasol.

La importacia de establecer las variaciones genotípicas y ambientales de la relación tegumento/embrión posibilitaría avanzar en el conocimiento de la determi- nación del porcentaje final de aceite del grano de girasol. Además, esta relación es interesante para el proceso industrial ya que el pericapio sometido a estrés térmico reduce la facilidad de descascarado del grano (Rondanini et al., 2006). Asimismo, ha sido reportado en diversos trabajos que la característica de la relación tegumento/ embrión (Khaleghizadeh, 2010) y bajo contenido de aceite (Mason et al.,1991) podría conferir algún tipo de protección contra el ataque de aves. Si bien se conoce por trabajos anteriormente publicados que, el porcentaje de tegumento del grano de girasol afecta el procentaje de aceite (Rondanini et al., 2003, 2009), en la actualidad no es clara la influencia de la proporción de tegumento en los genotipos modernos de girasol. La forma y tamaño de los granos podría explicar en parte las variaciones genotipicas en la relación tegumento/embrión. También es poco conocido el efecto ambiental sobre las fracciones de tegumento, embrión, la relación tegumento/embrión y el procentaje de aceite en regiones subtropicales del noreste de Argentina. En el presente trabajo se pretende estudiar el comportamiento de dichas características del grano de girasol en distintos genotipos con características agronómica contrastantes como así también la respuesta en diferentes ambientes del noreste Argentino.

\section{MATERIALES Y MÉTODOS}

\section{Descripción del sitio}

Se realizaron cuatro experimentos en la campaña 2014/2015. Los ensayos se llevaron a cabo en i) Agencia de Extensión Rural INTA Ceres (CER, 29 $9^{\circ} 84^{\circ} \mathrm{S}$ y $61^{\circ}$ $97^{\prime}$ O), ii) Villa Ocampo (VO, $28^{\circ} 25^{\prime} \mathrm{S}$ y $59^{\circ} 22^{\prime} \mathrm{O}$ ) y iii) Estación Experimental Agropecuaria INTA Reconquista $\left(29^{\circ} 08^{\prime} \mathrm{S}\right.$ y $59^{\circ} 38^{\prime} \mathrm{O}$ ) en dos fechas de siembra contrastante (Rqta 1 y 2, óptima y tardía, respectivamente). Las fechas de siembra fueron 9 de septiembre, 12 de agosto, 14 de agosto y 15 de octubre del 2014 para CER, VO, Rqta 1 y Rqta 2, respectivamente.

El experimento fue conducido en condiciones de secano (agua inicial al metro de profundidad 122, 218 y $200 \mathrm{~mm}$ en CER, VO, Rqta respectivamente) como parte de los ensayos comparativos de rendimiento de girasol perteneciente al programa nacional de cereales y oleaginosas de INTA, Proyecto nacional "Redes de evaluación de germoplasma inédito (INTA) y cultivares comerciales de Cereales y Oleaginosas", dentro del marco del convenio de vinculación tecnológica entre INTA y ASAGIR (PNCyO 1127046 convenio INTA-ASAGIR).

\section{Diseño estadístico y manejo del experimento}

En CER el experimento se implantó sobre un suelo perteneciente a la unidad cartográfica CRO 15 4ws 47, complejo de suelo Argiudol Típico con capacidad productiva B2 media-baja. En VO, el experimento se implantó sobre un suelo perteneciente a la unidad 
cartográfica RTA 14 4/5 ws (e)36 con una capacidad productiva B2- media baja; mientras que en Rqta el suelo perteneció a la unidad cartográfica RTA 043 ws59 , complejo de suelos donde el subgrupo Arguidol acuértico es dominante (Giorgi et al., 2009) y una capacidad productiva B1- media alta. Previo a la implantación del cultivo se llevó a cabo la extracción de muestras de suelo a 18 $\mathrm{cm}$ de profundidad para conocer

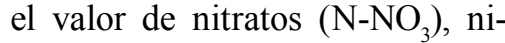
trógeno total ( $\mathrm{N}$ total), fósforo $(\mathrm{P})$ y materia orgánica (M.O). CER tiene $\mathrm{pH}$ neutro, mientras que Rqta 1, Rqta 2 y VO es moderadamente ácido.

Los tratamientos consistieron en una combinación de cuatro ambientes en donde se evaluaron 15 híbridos comerciales de diferentes empresas que se encuentran en el mercado actualmente. Los híbridos se seleccionaron en base a su importancia en el mercado y características agronómicas contrastantes (Tabla 1). En cada ambiente se sembraron los híbridos de girasol en cuatro (4) repeticiones. Las parcelas consistieron en 4 surcos de $5 \mathrm{~m}$ de largo, bajo un diseño experimental Alfa látice. El ensayo se sembró en siembra directa, la densidad utilizada fue de 4,5 a 5 pl. $\mathrm{m}^{-2}$, con una profundidad de siembra de $3 \mathrm{~cm}$. En el estado fenológico V4 se procedió al raleo de plantas para ajustar a la densidad deseada. Se controlaron malezas en forma manual y las plagas en forma química según umbral de daño durante el ciclo del cultivo.

\section{Determinaciones}

En los ensayos se registró el estado fenológico de emergencia, R5 y R9 (Schneiter y Miller, 1981) y se estimó los días a floración y madurez fisiológica por híbrido y localidad. Se recopilaron datos diarios de temperaturas (máximas, mínimas y media), radiación solar global y precipitación en cada localidad donde se realizaron los ensayos. La radiación global (RG) incidente se estimó diariamente a partir de heliofanía diaria. La radiación fotosintéticamente activa (PAR) incidente sobre las plantas se calculó como 0,48 x RG (Dosio et al., 2000).

Se determinó el tamaño del grano (largo y ancho, medido con calibre), peso de grano (en $\mathrm{mg}$ ), peso y espesor del tegumento, peso del embrión y porcentaje de aceite en grano y aceite del embrión (determinado con un resonador magnético nuclear, RMN, Spinlock,
Córdoba, Argentina). La relación tegumento/embrión se estimó a partir de la proporción del embrión respecto al pericarpio. La proporción del embrión en el grano se calculó como PE/(PT + PE), donde PE y PT representan el peso del embrión y tegumento, respectivamente (Izquierdo et al., 2008).

\section{Análisis de datos}

Los datos se analizaron utilizando la técnica del análisis de las varianzas en el software Infostat (Di Rienzo et al., 2013). Las diferencias entre híbridos por localidad se analizaron mediante el test de comparación de media (Tukey, $\alpha=0,05$ ). Se estimó la contribución relativa de los efectos híbridos $(\mathrm{H})$, ambiente (A) y su interacción (GxA), mediante el análisis de componentes de varianzas. Para determinar la estabilidad de los híbridos frente a distintos ambientes se utilizó el método de Shukla (Shukla, 1972).

Para generar variables ambientales y evaluar su efecto sobre las variables de grano recolectadas se calculó: 1- Período de determinación del número de granos: desde estado estrella a plena floración (R1-R5), 2Período de llenado de granos: desde plena floración a madurez fisiológica (R5-R9) y 3- Período 1 y 2: desde estado estrella a madurez fisiológica (R1-R9). Se calculó el promedio por período para temperatura máxima (TMX), media (TM), mínima (TMN) y la Radiación PAR incidente acumulada. Las precipitaciones se analizaron durante todo el período. Se realizaron análisis de componentes principales de la matriz de datos es- 
tandarizados para explicar la manera que se relaciona cada componente principal con las variables y el efecto que estas tienen, positivo o negativo dependiendo el ángulo de separación entre las mismas. Además se usaron gráficos del tipo biplots (Gabriel, 1971) para la representación gráfica de correlaciones entre variables determinadas de cultivo y grano con las variables fenológicas y climáticas. Con el fin de identificar las variables fenológicas y climáticas de mayor contribución en la explicación de las variaciones encontradas así como para cuantificar su impacto se utilizaron modelos de regresión lineal múltiple (Draper y Smith, 1998). La selección de los modelos de regresión de mejor ajuste fue realizada mediante el procedimiento stepwise de selección de variables (Draper y Smith, 1998). Se analizó la pendiente $=1(\mathrm{p}<0,05)$ con el programa IRENE (Integrated Resources for Evaluating Numerical Estimates, Beta Versión 1.03, Fila et al., 2003). Los análisis se realizaron mediante el uso de software Infostat (Di Rienzo et al., 2013) y/o el software Sigmaplot 8.0.
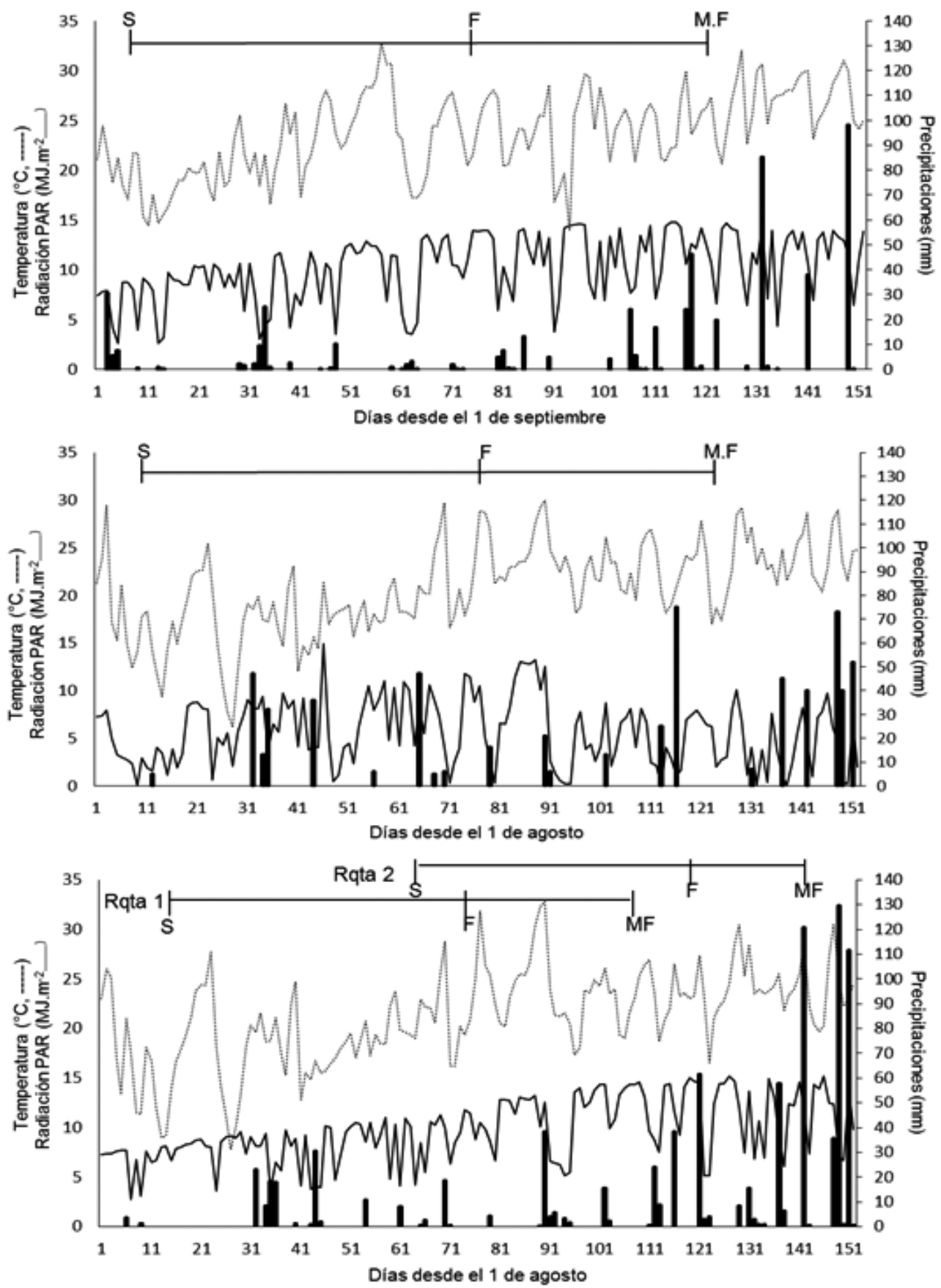

Figura 1. Temperatura media (TM), Radiación fotosintéticamente activa (Radiación PAR MJ.m² ${ }^{-2}$ y precipitaciones (mm) durante la campaña 2014-2015 en función de los días desde el a) 1 de septiembre en CER (Ceres) y 1 de agosto en b) VO (Villa Ocampo), c) Rqta 1 y Rqta 2. Las líneas horizontales en la parte superior de cada gráfico representan momento de siembra (S), floración (F) y madurez fisiológica (MF) aproximada. 


\section{RESULTADOS}

En CER, VO, Rqta 1 y Rqta 2 la máxima PAR interceptada fue 14, 15, 14 y 15 MJ.m ${ }^{-2}$, respectivamente. Las precipitaciones acumuladas en ciclo de cultivo fueron 505, 610, 450 y $351 \mathrm{~mm}$ en CER, VO, Rqta 1 y Rqta 2, respectivamente (Figura 1). La temperatura máxima durante el llenado de granos fue de $33,29,29$ y $31^{\circ} \mathrm{C}$ para CER, VO, Rqta 1 y Rqta 2, respectivamente, mientras que la temperatura mínima para dicho período fue de $18,18,17$ y $21^{\circ} \mathrm{C}$ para las localidades mencionadas anteriormente respectivamente.

El período comprendido de siembra a floración tuvo una duración aproximada de $80 \pm 0,6 ; 81 \pm 0,2 ; 81 \pm 0,5$ y $70 \pm 0,3$ días, mientras que los días desde emergencia a madurez fisiológica fue de $116 \pm$ 0,$5 ; 125 \pm 0,19 ; 123 \pm 0,5 ; 97 \pm 0,5$ días correspondiente a CER, VO, Rqta 1 y Rqta 2 , respectivamente. Por otro lado, la altura de plantas fue significativamente diferente entre híbridos y ambientes $(\mathrm{p} \leq 0,0073)$. En las localidades de CER, VO, Rqta 1 y Rqta 2 la altura promedio entre híbridos fue de $148 \pm 2,151 \pm 2,143 \pm 1,147 \pm 2$ $\mathrm{cm}$, respectivamente.

\section{Forma de grano}

Los tratamientos aplicados generaron importantes variaciones en el largo y ancho de grano $(\mathrm{p} \leq 0.0001)$. El largo de los granos fue significativamente diferente entre ambientes y genotipos (86 y $17 \%$ de la varianza total, respectivamente, $\mathrm{P} \leq 0,0001$ ), mientras que no se encontraron diferencias significativas en la interacción GxA $(<1 \%$ de la varianza total, $\mathrm{P}=0,4593)$. En las localidades de CER, VO, Rqta 1 y Rqta 2 la mayor longitud de grano fue de $12,8 \pm 0,2$; $11,6 \pm 0,2 ; 11 \pm 0,1$ y $11,4 \pm 0,3 \mathrm{~mm}$, mientras que la menor longitud fue $10,5 \pm 0,2 ; 9,2 \pm 0,1 ; 9,1 \pm 0,3 \mathrm{y}$ $8,7 \pm 0,4 \mathrm{~mm}$, respectivamente (Figura 2).

En relación al ancho del grano, se observaron diferencias entre híbridos y ambientes $(\mathrm{P} \leq 0,0448)$. El $93 \%$ de la variabilidad fue ambiental, mientras que el $6 \%$ restante fue por el genotipo (Figura 2). El promedio de ancho fue 5,$9 ; 4,9 ; 4,8$ y $5,2 \mathrm{~mm}$ para las localidades de CER, VO, Rqta 1 y Rqta 2, respectivamente.

Para el espesor del tegumento no se detectaron diferencias significativas en la interacción GxA $(\mathrm{P}=0,1699)$ mientras que se diferenciaron entre genotipos y ambientes (27 y $67 \%$ de la varianza total respectivamente, $\mathrm{P} \leq 0,0001)$. En CER el $53 \%$ de los genotipos obtuvieron espesor mayor a $0,5 \mathrm{~mm}$, en
VO el $20 \%$ únicamente y Rqta 2 el $13 \%$, mientras que en Rqta 1 el 93\% de los híbridos obtuvieron valores menores a $0,5 \mathrm{~mm}$ de espesor.

\section{Peso de grano}

Los tratamientos aplicados generaron importantes variaciones en el peso del grano y sus respectivas proporciones de tegumento y embrión $(\mathrm{P} \leq 0.0001)$. El peso de grano se diferenció entre genotipos (3\%), ambientes $(96 \%)$ y su interacción GxA $(1 \%, \mathrm{P}<0,0001)$. El intervalo de peso de grano en CER fue mayor que en los demás ambientes, entre 60,8 y $91 \mathrm{mg}$ (Figura 3). En VO la variación fue entre 37,5 y $58,2 \mathrm{mg}$. El menor peso en Rqta 1 y 2 fue 35,4 y 28,7 mg, respectivamente. El promedio para CER, VO, Rqta 1 y Rqta 2 fueron 75,$6 ; 48,68 ; 42,79$ y $38,69 \mathrm{mg}$, respectivamente. Las diferencias encontradas en el peso de grano se debieron 
a variaciones en el peso de tegumento, embrión y su contribución relativa. El peso de tegumento de los granos fue significativamente diferente entre ambientes, genotipos y la interacción GxA, $(\mathrm{P} \leq 0,0006)$. El 86\% de la variabilidad fue debido al ambiente, mientras que el $13 \%$ fue genotípica y el $1 \%$ de la interacción. En CER se alcanzó el mayor peso de tegumento con $33,7 \pm 0,2$, seguido por VO $(23,2 \pm 1,5)$, Rqta $1(22,7$ $\pm 1,1)$ y Rqta $2(22,2 \pm 2,8 \mathrm{mg})$. El menor peso de tegumento para CER, VO, Rqta 1 y Rqta 2 fue $16,7 \pm 1,4,12,2 \pm 0,2,10,2$ $\pm 0,2$ y $9,7 \pm 0,2 \mathrm{mg}$, respectivamente. Por otro lado, el peso del embrión también se observaron diferencias significativas en la interacción GxA ( $\mathrm{P}<0,0001)$. El 96\% de la variabilidad fue debido al ambiente, el $3 \%$ al genotipo y el $1 \%$ restante a la interacción GXA. CER es el ambiente con mayor peso del embrión en comparación con los tres ambientes restantes. En esta localidad el híbrido DK 3970 CL alcanzó el mayor peso $(81,2 \pm 6,1 \mathrm{mg})$. En cuanto al porcentaje del embrión del grano también se encontraron diferencias significativas en la interacción GxA $(\mathrm{P}<0,0158)$ obteniendo un rango entre $80,2 \pm 0,5$ y $62 \pm 3,4 \%$ (Figura 3 ). En las localidades de CER, VO y Rqta 1, el máximo porcentaje que se registró fue 80,2 $\pm 0,5 ; 76,5 \pm 2,8 ; 77,7 \pm 1,2 \%$, respectivamente, mientras que para el ambiente Rqta 2 fue de $76,0 \pm 3,3 \%$. Los promedios para CER, VO, Rqta 1 y Rqta 2 fueron 72,2; 71,$03 ; 71,08$ y $68,08 \%$, respectivamente. El $52 \%$ de la variabilidad fue debido al genotipo, mientras que el 46 y $2 \%$ debido al ambiente y a la interacción.

Las interacciones significativas para las variables peso de grano, tegumento y embrión indicaron que en CER los valores fueron mayores que en comparación a los demás ambientes. Como las diferencias no fueron constantes entre híbridos, se realizó un análisis Shukla para detectar la estabilidad de los mismos (Figura 4). Los genotipos 8, 13, 14 y 15 obtuvieron el mayor peso de grano y fueron los más estables. Por otro lado, los híbridos 2, 6 y 10 tuvieron un peso de granos promedio superior a la media pero fueron menos estables en los distintos ambientes. Aquellos cultivares que presentaron baja variación ambiental y un elevado peso de grano también demostraron tener mayor peso de embrión y menor peso de tegumento manteniendo la estabilidad, esto se debe a que el peso del embrión explica en gran parte al peso del grano total.
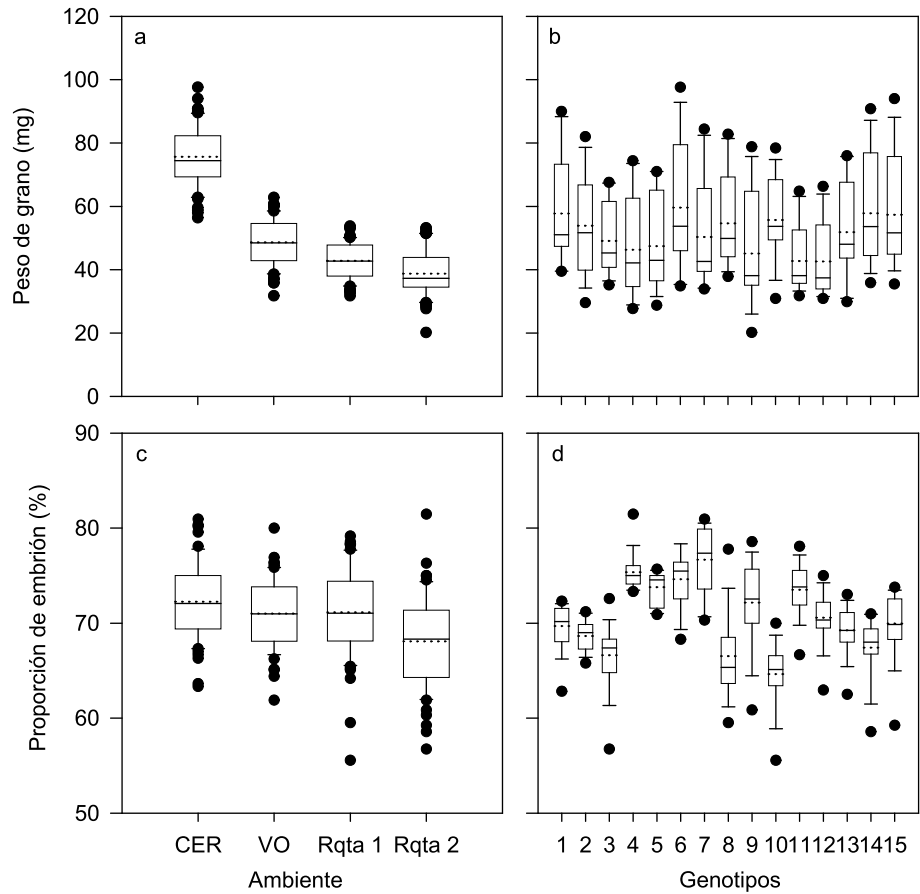

Figura 3. Gráfico de cajas correspondiente al peso de grano (mg, a y b) y proporción de embrión (\%, c y d) cuatro ambientes (a y c) y los híbridos comerciales de girasol (b y d) durante la campaña 2014/15. Líneas punteadas y continuas corresponden al promedio y mediana de la serie.

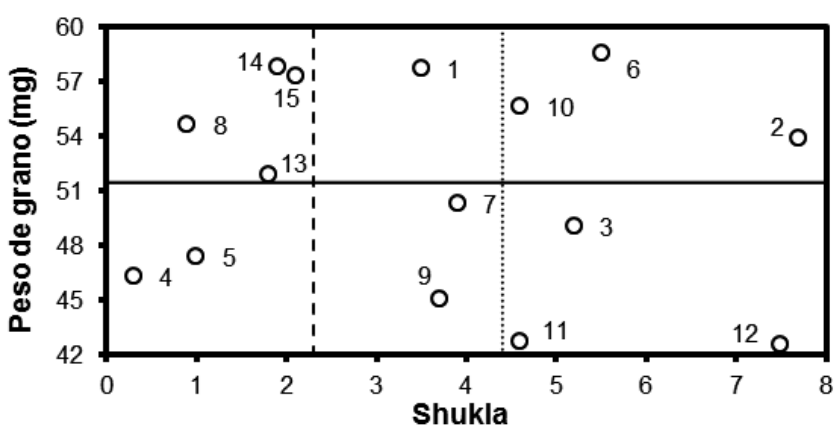

Figura 4. Análisis de estabilidad de los híbridos (ID del híbrido, Cuadro 2) para el peso de grano en función del coeficiente Shukla. Línea horizontal representa la media del peso de grano. Las líneas verticales segmentada y de puntos indican estabilidad significativa al $\mathrm{P}<0,01$ y $\mathrm{P}<0,05$ respectivamente.

\section{Porcentaje de aceite}

El aceite en el grano se diferenció estadísticamente entre genotipos, ambientes y la interacción GxA $(17,71$ y $11 \%$ de la varianza total respectivamente, $\mathrm{P} \leq 0,0001$, Figura 5). El promedio por localidad fue $50 \%$ para CER, VO, Rqta 1 y $48 \%$ para Rqta 2 , respectivamente. El 91\% de la variabilidad en el aceite del embrión fue debido al ambiente, mientras que 6 y $3 \%$ se debieron al efecto del genotipo y la interacción $(\mathrm{P}<0,0001)$. En CER, el mayor porcentaje de aceite fue $66,0 \pm 0,3 \%$, mientras que el menor porcentaje de aceite fue de $54,7 \pm 0,7$. En VO los porcentajes varia- 

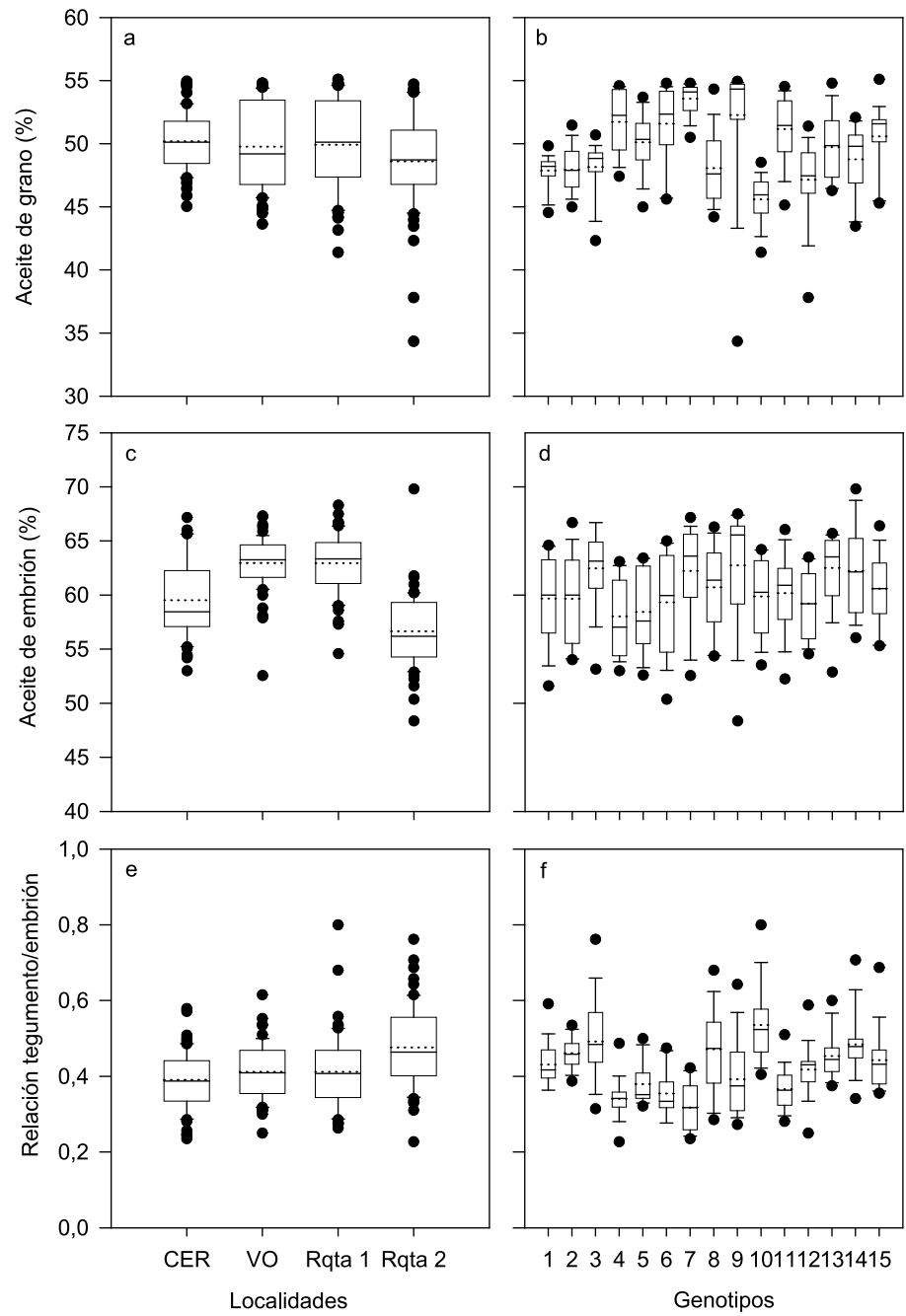

Figura 5. Gráfico de cajas correspondiente al aceite de grano $(\%$, a y b), aceite del embrión (\%, c y d) y relación tegumento/ embrión (e y f) en cuatro ambientes (a, c y e) y los híbridos comerciales de girasol (b, d y f) durante la campaña 2014/15. Líneas punteadas y continuas corresponden al promedio y mediana de la serie.

ron entre $66,3 \pm 0,6$ y $59,8 \pm 0,6 \%$. Para el ambiente de Rqta 1, el rango de aceite del embrión estuvo comprendido entre $65,60 \pm 0,6$ y $60,4 \pm 2,1 \%$. En cuanto a Rqta 2, la variación fue de $60,4 \pm 3,1 \%$ y $53,5 \pm$ $1,0 \%$ (Figura 5). En promedio, en los ambientes de VO y Rqta 1, se obtuvo un porcentaje de aceite en el embrión del 63\%, mientras que en CER fue de $60 \%$ y Rqta 2 de 57\%. Por último, la variabilidad la relación tegumento/embrión por ambiente, genotipo y GXA fue 53, 40 y $7 \%$, respectivamente $(\mathrm{P} \leq 0,0007$, Figura 5). En CER, Rqta 1 y VO el híbrido que obtuvo menor relación tegumento/embrión fue de $0,25 \pm 0,004$, $0,29 \pm 0,01$ y $0,35 \pm 0,02$, respectivamente. En Rqta 2 , la menor relación $\mathrm{T} / \mathrm{E}$ fue de $0,32 \pm 0,03$.

\section{Relaciones entre las variables}

En el análisis de componentes principales (Figura 6) los ambientes están representados por círculos mientras que las variables por vectores. La sumatoria de ambas componentes (CP1 + CP2) explican el $67,1 \%$ de la variación total. La CP1, es la componente que explica el $39,1 \%$ de la variabilidad total y separa los ambientes de CER (a la izquierda) de Rqta 2 (derecha). El CP2 explica el $28,0 \%$ de la variabilidad total. Por debajo de la línea 0 se encuentran en un mismo sector Rqta 1 y VO, las CP1 y 2 no separaron estos ambientes lo que indica que son similares con los híbridos analizados. Como es de esperar las temperaturas y radiaciones estuvieron correlacionadas dentro de cada período. Del mismo modo, el peso de grano estuvo relacionado con el peso de tegumento y embrión, el porcentaje de aceite de grano con el del embrión $\mathrm{y}$ el rendimiento con el número de granos. Los días a floración se relacionaron de forma positiva con los componentes del rendimiento, el aceite en grano y embrión, esto fue debido a cambios en las condiciones ambientales durante el período de que cada variable se define.

A partir de los resultados observados en el análisis de componentes principales, donde emergen claras correlaciones entre las variables, se ajustaron modelos de regresión múltiples, cada uno basado en el grupo de variables predictoras. Se encontró que la RT/E tiene relación negativa con el porcentaje de aceite en grano $(\mathrm{P}<0,0001$, Figura 7). La RT/E explicó el $40 \%$ de la variación del porcentaje de aceite de grano. Asimismo, DAF tienen efectos positivos sobre el aceite en el embrión, mientras que la TM R1R9 negativo. Éstas dos variables explican el $62 \%$ de la dispersión de los datos $(\mathrm{P}<0,0001$, Tabla 2). A su vez, la relación tegumento/embrión presentó relación positiva con el espesor de tegumento, precipitaciones durante el periodo y la DAF. El peso de grano, TMX R1R5 y el porcentaje de aceite en grano explicaron el $95 \%$ de la variabilidad del peso del embrión ( $\mathrm{P}<0,0001$, Tabla 2). En cuanto al peso de tegumento, fue explicado por el peso de grano, EC, precipitaciones y DAF en forma positiva, mientras que el $\%$ de aceite lo afecto de manera negativa $(\mathrm{P}<0,0001)$. El 90\% de la variabilidad de los pesos de grano fue explicado por los granos. $\mathrm{m}^{-2}$, las temperaturas máximas de R1 a R5 y el espesor de tegumento en forma positiva y de manera negativa las temperaturas medias de R1 a R5 $(\mathrm{P}<0,0001)$. El peso del tegumento explicó el $55 \%$ de la dispersión en EC. Para cada una de las variables analizadas, las pendientes no difirieron significativa- 
Tabla 2. Resumen del análisis estadístico (coeficientes de ajuste, $\mathrm{p}$-valor y $\mathrm{R}^{2}$ ) de las variables $\%$ aceite, $\mathrm{AE}$ (Aceite Embrión), R T/E (Relación tegumento/embrión), PE (Peso Embrión), PT (Peso Tegumento), PG (Peso de grano), ET (Espesor Tegumento), en función de los factores predictivos DAF (Días a Floración), TMR1R9 (Temperatura Media del ciclo), Prec (Precipitaciones), TMXR1R5 (Temperatura Máxima de floración).

\begin{tabular}{|c|c|c|c|}
\hline Variable & Factores y coeficientes de ajuste & p-valor & $\mathrm{R}^{2}$ \\
\hline$\%$ aceite & $53,81-22,83 *(R T / E)$ & $P<0,0001$ & 0,51 \\
\hline $\mathrm{AE}$ & 78,99-1,36*(TM R1R9)+0,15*(DAF) & $P<0,0001$ & 0,62 \\
\hline $\mathrm{R} T / \mathrm{E}$ & $0,65+0,00014 *(\operatorname{Prec})+0,27 *(\mathrm{ET})+0,0023 *(\mathrm{DAF})$ & $P<0,0001$ & 0,63 \\
\hline $\mathrm{PE}(\mathrm{mg})$ & $-55,89+0,75 *(P G)+1,42 *(T M X$ R1R5)+0,37(\%Aceite) & $P<0,0001$ & 0,95 \\
\hline PT (mg) & $-4,41+0,31 *(P G)+19,93 *(E T)+0,01 *($ Prec. $)-0,40 *(\%$ Aceite $)+0,20 *(D A F)$ & $P<0,0001$ & 0,90 \\
\hline PG (mg) & $-96,47+0,0046 *\left(\right.$ grano. $\left.m^{-2}\right)+2,12^{*}(\mathrm{TMX} \mathrm{R} 5 \mathrm{R} 9)+38,57^{*}(\mathrm{ET})+4,21^{*}(\mathrm{~L})+6,62^{*}(\mathrm{~A})-0,01^{*}(\operatorname{Prec})$ & $P<0,0001$ & 0,91 \\
\hline ET & $0,24+0,01 *(\mathrm{PT})$ & $P<0,0001$ & 0,55 \\
\hline
\end{tabular}

mente de $1(\mathrm{p}>0,05)$. Los modelos múltiples obtenidos estimaron satisfactoriamente los datos obtenidos a campo en cada localidad (Figura 7).

\section{DISCUSIÓN}

El tamaño de grano obtenido en el presente trabajo (tanto en ancho como en largo) fue diferente entre genotipos y ambientes. La ausencia de interacción GxA indica que es un parámetro genotípico estable entre ambientes. Asimismo, el mayor tamaño reportado en la localidad de Ceres (en el cual también se reportó el mayor peso de grano) indica que la fuente disponible para el llenado de granos fue mayor en esa localidad. Aumentos en la disponibilidad de asimilados durante el llenado de granos genera incrementos en el tamaño del grano pero dependerá del tamaño final del tegumento (Dosio et al., 1997) ya que ésta determina el tamaño final del embrión (Santalla et al., 2003). Esta diferencia de tamaño está principalmente debida a la tasa (Connor y Hall, 1997) y/o duración (Chimenti et al., 2001) del llenado de granos, mientras que también hay una gran diferencia entre híbridos. En estudios realizados por Santalla y Mascheroni (2003) determinaron que la longitud de los granos alto oleico variaron entre $10-12 \mathrm{~mm}$, mientras que en el ancho no hubo variación $(5-5,4 \mathrm{~mm})$. La longitud reportada en este trabajo fue similar a la obtenida en los ensayos realizados para híbridos convencionales, mientras que para la variable ancho el intervalo fue mayor $(6,6-4,2 \mathrm{~mm})$.

La variabilidad genotípica y ambiental para el peso del grano estuvo influenciada principalmente por las temperaturas máximas y mínimas desde R1 a R5, el número de granos por metro cuadrado y el

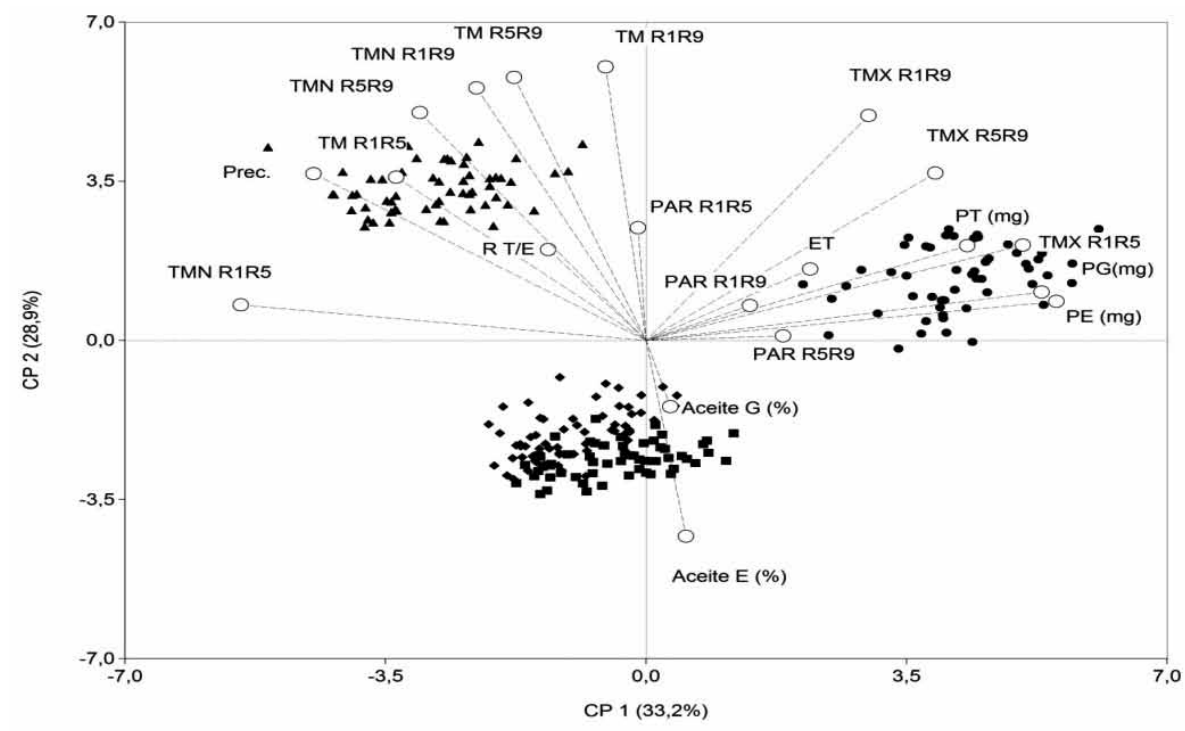

Figura 6. Biplot del análisis componentes principales, en donde los ambientes están representados por círculos, cuadrados, rombos y triángulos a CER, VO, Rqta 1 y Rqta 2, respectivamente. Los vectores son variables como aceite embrión (Aceite E, \%), aceite grano (Aceite G, \%), peso de grano (PG, mg), peso de embrión (PE, mg), peso de tegumento (PT, mg), espesor de tegumento (ET), relación tegumento/embrión (R T/E), Precipitaciones (Prec.) y radiación fotosintéticamente activa (PAR), temperatura máxima (TMX), temperatura media (TM), temperatura mínima (TMN) durante el periodo R1-R5 y R5-R9. 
espesor del tegumento. Dosio et al., (2000) y Otegui y López Pereyra, (2003) encontraron que retrasos en fecha de siembra inciden negativamente sobre el peso del grano, debido a que las plantas se exponen a temperaturas altas (Rondanini et al., 2007) y baja radiación durante llenado de grano (Aguirrezábal et al., 2003). Además, si ésta disminución ocurriera en la etapa inicial de llenado de grano afectaría el peso individual de los granos en los diferentes sectores del capítulo (Andrade y Ferreiro, 1997) decreciendo desde la periferia hacia el centro debido a una mayor oferta de nutrientes (Santalla et al., 2002). Por otro lado, a pesar que los híbridos estriados contienen menor proporción de embrión, el peso por grano de estos es mayor que los híbridos de tegumento negra (Izquierdo et al., 2008).

El peso del embrión estuvo relacionado con la temperatura máxima en R1 a R5, el peso del grano y el porcentaje de aceite. Chimenti et al., (2001) por otro lado, observaron que la temperatura tiene un marcado efecto sobre el peso final del embrión, modificando tanto la duración de la fase como la tasa de llenado de la misma. Asimismo Gesch y Johnson, (2012) reportaron que al aumentar el contenido de aceite, la proporción de embrión aumenta, existiendo una correlación directa entre estas dos variables. Por último la proporción del grano ocupado por el embrión varió entre un 62 a $80 \%$ similar a lo que lo reportado por Aguirrezábal et al., (1996). Recientemente Castillo et al., (2017) encontraron asociaciones positivas entre el peso de grano y peso de ovario en R3, en antesis (R5), tasa de crecimiento de ovario, dimensiones de grano y humedad de grano. Similar a lo reportado en el presente trabajo, en las primeras etapas de crecimiento de los ovarios durante el período de pre-antesis y una reducción de la fuente en pre-antesis afectó el peso de grano y la concentración de aceite de grano. En el presente trabajo, el peso del tegumento estuvo relacionado con su espesor, el peso individual del grano, los días a floración (donde se define las reservas para el llenado del grano) y las precipitaciones. En fechas tardías, al acortarse el período de llenado del embrión, la proporción de tegumento en el grano es mayor, lo que lleva a que la relación tegumento/embrión aumente (Killi y Altunbay, 2005). En girasol, el tegumento es un componente importante en el peso del grano en que varía entre 12 y 35\% (Connor y Sadras, 1992; Sadras y Villalobos, 1994; Denis y Vear, 1996; Rondanini et al., 2007; Gesch y Jonhson; 2012). Asimismo, Ploschuk y Hall (1995) y Rondanini et al., (2007) reportaron variabilidad genética y ambiental tanto en la proporción de embrión como en la tegumento. Estas diferencias se debieron a efectos sobre la duración del llenado del grano y la duración de la floración lo que concuerda con los resultados de los análisis multiambientales realizados en este trabajo.
La concentración de aceite en el grano varía entre 48 y $54 \%$, mientras que en el embrión el porcentaje esta reportado entre $65-70 \%$ (López Pereira et al., 1999). Los resultados obtenidos tanto en aceite
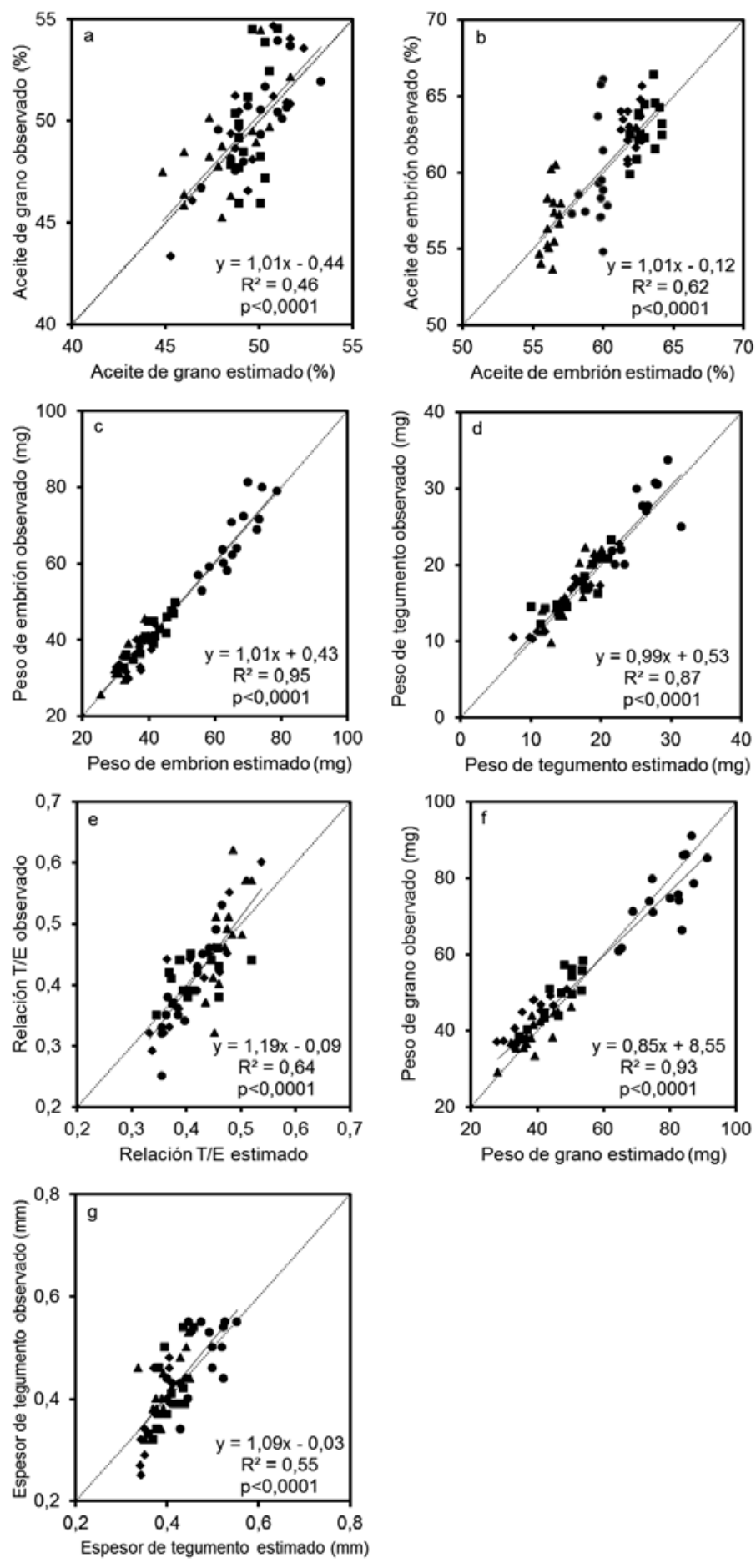

Figura 7. Relación entre datos observados versus estimados a partir de las ecuaciones obtenidas (tabla 2) de aceite de grano $(\%, a)$, aceite del embrión (\%, b), peso de embrión (mm, c), peso de tegumento $(\mathrm{mm}, \mathrm{d})$, relación T/E (e), peso de grano $(\mathrm{mg}, \mathrm{f})$ y espesor de tegumento (mm, g). Rombos, círculos, cuadrados y triángulos corresponden a CER, VO, Rqta 1 y Rqta 2. Línea punteada representa la relación 1:1. 
en grano (43 y $55 \%$ ) como en embrión (entre 53 y $66 \%$ ) fueron similares a los valores anteriormente mencionados. De la Vega y Hall (2002) encontraron menores rendimientos de aceite en fechas tardías en comparaciones con siembras tempranas por menor radiación solar interceptada (Izquierdo et al., 2008) y menor duración del llenado de granos (Rondanini et al., 2006). Además, Izquierdo et al., (2008) afirmaron que una reducción de la radiación interceptada provoca disminución en el porcentaje de aceite tanto en híbridos de tegumento estriadas como negra, pero estos últimos son más sensibles a variaciones en la radiación. Por lo tanto, híbridos estriados podrían ser considerados para siembras tardías. Sin embargo, las variaciones en el contenido de aceite en la semilla es causado por cambios en la proporción del peso de tegumento, peso del grano, concentración de aceite en tegumento y las variaciones en el contenido de aceite del embrión (Dosio et al., 2000). Por otra parte, Aguirrezábal y Pereyra (1998) observaron que un mismo genotipo sembrado en diferentes ambientes puede tener variaciones de un $10 \%$ por encima del promedio en el rendimiento de aceite en grano. En este trabajo se reporta una variación total de $12 \%$ entre híbridos y ambientes, de las cuales el $71 \%$ de dicha variación se debió al efecto ambiental. El porcentaje de aceite en grano se relacionó negativamente con la relación tegumento/embrión, por lo que el ambiente afecta principalmente el porcentaje de aceite del embrión (De la Vega y Hall, 2002) y las proporciones de tegumento y embrión. La diferencia entre híbridos y ambientes en el aceite del embrión fue explicado no solo por los días a floración, sino también por la temperatura durante el período reproductivo. Esto concuerda con Seiler (1986) quién observó que el contenido de aceite se asocia negativamente con la temperatura tanto en ambientes controlados como en ensayos a campo. López Pereira (1999) encontró que las diferencias entre híbridos para porcentaje de aceite son explicadas por la tasa de acumulación de aceite, por lo cual está asociado a la temperatura durante el llenado de granos. Por otra parte, Izquierdo et al., (2008) afirmaron que la concentración de aceite en el embrión es afectada por la radiación interceptada y que la mayor concentración de aceite en híbridos de tegumento negra en comparación a los estriados se debió a una mayor proporción en el embrión. Por lo tanto, los híbridos en la fecha tardía tuvieron mayor proporción de tegumento y menor aceite en el embrión que generaron importantes reducciones en el porcentaje de aceite de grano. El mejoramiento genético en híbridos de girasol con alto contenido de aceite llevó a una reducción en el tamaño de la semilla, el porcentaje de tegumento y una disminución de la relación tegumento/embrión (Denis et al., 1994). Los híbridos que producen alta concentración de aceite usualmente tienen tegumento negra, mientras que con baja concentración de aceite son de tegumento estriado. Esto se debe a que, los híbridos estriados tienen menor proporción de embrión en el grano (Izquierdo et al., 2008). Además, Santalla et al., (2003), reportaron que la relación embrión/ grano se incrementa desde la periferia hacia el centro del capítulo, no siendo afectada por las variaciones de niveles de radiación interceptada en híbridos de alto potencial en porcentaje de aceite, mientras que ocurre lo opuesto en genotipos de bajo potencial.

Conocer el comportamiento genotípico de los híbridos modernos de girasol en distintas condiciones ambientales es una herramienta fundamental de manejo que permitiría optimizar la producción de aceite y adecuar prácticas culturales. Poder determinar la estabilidad y/o adaptabilidad es fundamental para caracterizar los híbridos presentes en el mercado así como también de aquellos nuevos genotipos producidos por los diferentes planes de mejoramiento de Argentina. Por otra parte, conocer el porcentaje de aceite del embrión es una herramienta clave para el mejoramiento genético con el fin de obtener genotipos superiores en productividad de aceite sin comprometer el rendimiento de los mismos. Una aplicación fundamental de esta temática lo constituye el tamaño de grano potencial usado como herramienta para reducir el daño por aves. Las aves, por ejemplo palomas, afectan al cultivo principalmente cuando los granos de girasol ya están desarrollados, siendo en ocasiones estos daños considerables. Los daños por aves, si bien han sido poco evaluados en nuestro país, pueden ser importantes y constituir la limitante principal de la producción de este cultivo en ciertas regiones (Canavelli, 2010). Además los granos de tamaños más grandes (Bernardos y Farrell, 2012) y estriados (Zuil y Colombo, 2012) son menos afectados por daños de palomas. En este trabajo se comprobó que, de los genotipos analizados, el incremento de tamaño por mejores condiciones ambientales fue de 11 y $20 \%$ en largo y ancho de grano, respectivamente. Futuros trabajos se deberían enfocar en otros genotipos (no aceiteros) con una mayor plasticidad en tamaño de granos que aporten a esta problemática y considerando las proporciones de tegumento, embrión y porcentaje de aceite del embrión. No obstante, los datos encontrados en relación al porcentaje de aceite de tegumento, embrión y grano constituyen un insumo fundamental para la comparación con los genotipos presentes en el mercado actual.

\section{CONCLUSIÓN}

Se determinó una importante variabilidad tanto genotípica y ambiental (aunque esta variabilidad no fue interactiva) en la forma de los granos. Existen importantes diferencias entre genotipos y ambientes en cuanto al largo de grano, ancho de grano y espesor de 
tegumento, por lo que las diferencias dependen del híbrido y localidad que se analice. Se obtuvo mayor espesor de tegumento en ambiente con mejor rendimiento. Asimismo, en el porcentaje final de aceite del grano se demostró una alta variabilidad genotípica. La misma pudo explicarse por variaciones del aceite del embrión, por tener mayor efecto en comparación con el aceite del tegumento. Tanto el ambiente como el genotipo influencian la relación tegumento/ embrión y por ende esta característica del grano se correlaciona negativamente con el aceite final del grano y directamente relacionada con el espesor de tegumento. El análisis de componentes principales permitió identificar las variables climáticas que mejor explican las variaciones en rendimiento, porcentaje de aceite en el grano y proporción de tegumento y embrión. Además de las diferencias genéticas de los híbridos evaluados, se lograron detectar variables ambientales que explicaron parcialmente la respuesta de los híbridos. Los factores ambientales más importantes y frecuentes que se relacionaron con las variables analizadas fueron la TMXR1R5 y TMR1R9.

\section{AGRADECIMIENTOS}

Los autores agradecen la ayuda financiera otorgada a través del Proyecto PNCYO 1127046 "Redes de evaluación de germoplasma inédito (INTA) y cultivares comerciales de Cereales y Oleaginosas" y SANFE 1261309 "Aportes al desarrollo con enfoque territorial en el Domo Oriental e Islas del norte de Santa Fe". Los autores también desean agradecer a la Ing. Ana María Brach por la revisión del presente manuscrito y al Sr. Claudio Lorenzini por la asistencia técnica en los ensayos.

\section{BIBLIOGRAFIA}

Aguirrezábal, L; Orioli G; Hernandez, L; Pereyra, V; Miravé, J. 1996. Aspectos fisiológicos que determinan el rendimiento. Unidad Integrada Balcarce. Offset Vega.

Aguirrezábal, L; Pereyra, V. 1998. Girasol. En: Aguirrezábal, L.; Andrade, F (Eds.). Calidad de productos agrícolas. Bases ecofisiológicas, genéticas y de manejo agronómico. Unidad Integrada Balcarce, 140197.

Aguirrezábal, L; Lavaud, Y; Dosio, G; Izquierdo, N; Andrade, F; Gonzalez, L. 2003. Intercepted Solar Radiation during Seed Filling Determines Sunflower Weight per Seed and Oil Concentration. Crop Sci.43:152-161.

Andrade, F; Ferreiro, M. 1997. Reproductive growth of maize, sunflower and soybean at different source levels during grain filling. Field Crops Res 48, 155-165.

Bernardos, J; Farrell, M. 2012. Evaluación de daño por paloma torcaza (Zenaida auriculata) en girasol y perdida de cosecha en la provincia de La Pampa. Ediciones INTA.
Canavelli, S. 2010. Consideraciones de manejo para disminuir los daños por aves en girasol. Información técnica cultivos de verano. Campaña 2010. Publicación miscelánea $\mathrm{n}^{\circ} 118$.

Castillo, F.M.; Vázquez, S.C; Calderini, D.F. 2017. Does the pre-flowering period determine the potential grain weight of sunflower? Field Crops Research 212: $23-33$

Chimenti, C; Hall, A. 2001. Grain number responses to temperature during floret differentiation in sunflower. Field Crops Res. 72: 177-184.

Connor D; Hall, A. 1997. Sunflower physiology. Sunflower technology and production. Agronomy monograph $\mathrm{N}^{\circ} 35$. American society of agronomy, crop science society of America. Pp 113-181.

Connor, D; Sadras, V. 1992. Physiology of yield expression in sunflower. Field Crops Res. 30: 333-389.

De La Vega, A; Hall, A. 2002. Effects of planting date, genotype, and their interactions on sunflower yield: II. Components of oil yield. Rop sci. 42:1202-1210.

Denis, L; Dominguez, J; Baldini, M; Vear, F. 1994. Genetical studies of hullability in comparison with other sunflower seed characteristics. Euphytica. 79, Issue 1, pp 29-38.

Denis, L; Vear, F. 1996. Variation of hullability and other seed characteristics among sunflower lines and hybrids. Euphytica 87: 177 -187.

Di Rienzo, J; Casanoves, F; Balzarini, M; Gonzalez, L.; Tablada, M; Robledo, C. Infostat versión 2013. Grupo infostat, FCA, Universidad Nacional de Córdoba, Argentina. Disponible en: http://www.infostat.com.ar.

Dosio, G; Aguirrezábal, L; Andrade, F; Pereyra, V. 2000. Solar ratiation intercepted during seed filling and oil production in two sunflower hybrids. Crop Sci. 40:16371644.

Dosio, G; Izquierdo, N; Aguirrezábal, L. 1997. La iPAR afectó la dinamica de llenado y de acumulación relativa de aceite en frutos de girasol del híbrido Dekalb G100. Rev. Facultad de Agronomia. 17: 117-122.

Ddraper, N; Smith, H. 1998. Applied Regression Analysis (3rd edition). New York: Wiley 736 pp.

Figueiredo, A; Baumler, E; Riccobene, I; Nolasco, S. 2011. Moisture-dependent engineering properties of sunflower seeds with different structural characteristics. Journal of Food Engineering 102. 58-65.

Gabriel, K. 1971. The biplot graphic display of matrices with application to principal component analysis. Biometrika 58: 453-467.

Gesch, R; Johnson, B. 2012. Seed moisture at physiological maturity in oilseed and confectionary sunflower hybrids in the northern U.S. Field Crops Res. 133, 1-9.

Giorgi, R; Tosolini, R; Sapino, V; eon C; Chiavassa, A. 2009. Capacidad productiva de los suelos de la provincia de Santa Fe. INTA-CR Santa Fe, EEA Rafaela.

Izquierdo, N; Dosio, G; Cantarero, M; Lujan, J; Aguirrezábal, L. 2008. Weight per grain, oil concentration, and 
solar radiation intercepted during grain filling in black hull and striped hull sunflower hybrids. Crop science 48:688-699.

Khaleghizadeh, A. 2010. Effect of morphological traits of plant, head and seed of sunflower hybrids on house sparrow damage rate. Crop Protection 30: 360-367.

Killi, F; Altunbay, S. 2005. Seed Yield, Oil Content and Yield Components of Confection and Oilseed Sunflower (Helianthus annuus L.) Cultivars Planted in Different Dates. International journal of agriculture and biology 1: $21-24$

Lindström, L.I; Hernández, L.F. 2015. Developmental morphology and anatomy of the reproductive structures in sunflower (Helianthus annuus): a unified temporal scale. Botany 93(5): 307-316, 10.1139/cjb-2014-0245

López Pereira, M; Rondanini, D; Trapáni, N. 2006. Girasol. En: De La Fuente, E. B; Gil, A; Gimenez, P; Kantolick, A; López Pereira, M; Ploschuk, E; Sorlino, D; Vilariño, M; Wassner, D; Windauer, L. Cultivos industriales. Buenos Aires. Facultad de Agronomía Universidad de Buenos Aires. Pp. 145-174.

López Pereira, M; Trapáni, N; Sadras, V. 1999. Genetic improvement of sunflower in Argentina between 1930 and 1995. II. Phenological development, growth and source -sink relationship. Field Crops Res. 63, 247-254.

Mason, J; Nuechterlein, G; Linz, G; Dolbeer, R; Otis, D. 1991. Oil concentration differences among sunflower achenes and feeding preferences of red-winged blackbirds. Crop Protection Vol: 10

Otegui, M; López Pereyra, M. 2003. Fecha de siembra. Capítulo 12. En: Producción de Cultivos de Grano. Bases funcionales para su manejo. (Ed. Pascale, A.J.) Editorial de la Facultad de Agronomía. Universidad de Buenos Aires.783 pp.

Ploschuk, E; Hall, A. 1995. Capitulum position in sunflower affects grain temperature and duration of grain filling. Field Crops Res. 44:111-117.

Rondanini, D; Mantese, A; Savin, R; Hall, A. 2006. Responses of sunflower yield and grain quality to alternating day/night high temperature regimes during grain filling: effects of timing, duration and intensity of exposure to stress. Field crops research 96 48-62.
Rondanini, D; Savin, R; Hall, A. 2003. Dynamics of fruit growth and oil quality of sunflower exposed to brief intervals of high temperature during grain filling. Field Crops Res. 83, 79-90.

Rondanini, D; Savin, R; Hall, A. 2007. Estimation of physiological maturity in sunflower as a function of fruit water concentration. European Journal of Agronomy 26: 295-309.

Rondanini, D.P; Mantese, A; Savin, R; Hall, AJ. 2009. Water content dynamics of achene, pericarp and embryo in sunflower: associations with potential achene size and its dry-down. European Journal of Agronomy 30: 53-62

Sadras, V; Villalobos, F. 1994. Physiological Characteristics Related to Yield Improvement in Sunflower (Helianthus annuus L.). Genetic Improvement of Field Crops. 287-320.

Santalla, E; Mascheroni, R. 2003. Note: Physical Properties of High Oleic Sunflower Seeds. Food Sci Tech Int. 435- 442

Santalla, E; Dosio, G; Nolasco, S; Aguirrezábal, L. 2002. The effects of intercepted solar radiation on sunflower seed composition from different head positions. JAOCS, vol. 79 (1): 69-74.

Schneiter, A; Miller, J; 1981. Description of sunflower growth stages. Crop Science. 21: 901-903

Seiler, G. 1986. Analysis of their relationships of enviromental factors with seed oil and fatty concentration of wild annual sunflower. Field Crops Res. 15:57-72.

Sharma, R; Sogi, D; Saxena, D. 2008. Dehulling performance and textural characteristics of unshelled and shelled sunflower (Helianthus annuus L.) seeds. Journal of Food Engineering 92: 1-7.

Shulka, G. 1972. Some statistical aspects of partitioning genotype-environmental components of variability. Pp 237-245.

Zuil, S., Colombo, F. 2012. Influence of morphological head traits of sunflower on "eared dove" (Zenaida auriculata) depredation. 18th International Sunflower Conference Proceeding. 07-VC-4: 887-892. 